\title{
EVALUATING STUDENT MOTIVATION AND PRODUCTIVITY DURING MAPATHONS
}

\author{
C. Green ${ }^{1}$, V. Rautenbach ${ }^{1, *}$ and S. Coetzee ${ }^{1}$ \\ ${ }^{1}$ Centre for Geoinformation Science, Department of Geography, Geoinformatics and Meteorology, University of Pretoria, \\ Pretoria, South Africa - cameron.green@tuks.co.za, (victoria.rautenbach, serena.coetzee)@ up.ac.za
}

Commission IV, WG IV/4

KEY WORDS: OpenStreetMap, OSM, mapathons, volunteer motivation, volunteered geographic information, VGI

\begin{abstract}
:
During mapathons, volunteers from various backgrounds get together to map a specific area using satellite imagery or aerial photographs. The expertise and motivation of these volunteers generally differ. In this paper, we present our results from an evaluation of university students' motivation for participating in mapathons and their productivity (i.e. how much data they contributed). To achieve our aim, we hosted four mapathons for final year university students where the participants were asked to complete a short questionnaire to determine their motivations and personal opinions of the mapathon. Afterwards, the productivity for two mapathons was evaluated. Participants indicated that they felt a sense of humanitarianism by contributing to communities in need. Additionally, the social aspect came through with a large percentage of the participants indicating that mapathons are fun and that they learned something new, for example, by improving their digitizing skills or that humanitarian organizations need help. Participants also indicated that the tools (i.e. OSM and iD editor) were easy to use, but that the imagery is sometimes not good enough due to cloud coverage. The general productivity for two mapathons was evaluated and we found that with more experience the participants were generally more productive. The results from this evaluation provided insight and knowledge that can assist mapathon organisers to create a productive environment for participants with the hopes of encouraging the participants to produce high quality data.
\end{abstract}

\section{INTRODUCTION}

A mapathon (literally "map marathon") is a collaborative effort, usually performed by groups of people who aim to collect specific map data through remote mapping (typically for humanitarian purposes) in places where OSM data is scarce or non-existent (Coetzee et al. 2018). With organizations, such as Missing Maps or YouthMappers, there are numerous mapathons being held monthly to assist the mapping efforts for various humanitarian projects. However, limited information is available about the motivation of the participants, or their perception of the mapathons.

In this paper, we present our results from an evaluation of university students' motivation for participating in mapathons and their productivity (i.e. how much data they contributed). For this evaluation, we hosted four mapathons for final year university students where the participants were asked to complete a short questionnaire to determine their motivations and personal opinions of the mapathon. The results from this evaluation provide insight and knowledge that could assist mapathon organisers to create a more productive environment for participants with the hopes of encouraging them to produce high quality data. The remainder of the paper is structured as follows: in Section 2 we briefly discuss the method followed; results are presented in Section 3; and in Section 4 the results are discussed, and conclusions are drawn.

\section{METHOD}

\subsection{Overview}

We hosted four mapathons for final year students enrolled for a geoinformatics module at the University of Pretoria, South Africa, to determine their motivations and opinions of the mapathon. Table 1 provides an overview of the mapathons.

The process followed during each mapathon was very similar. At the beginning of the mapathon, the participants were welcomed, and a short introduction was given on OpenStreetMap (OSM) and how to map features in OSM with iD editor. The TeachOSM (https://tasks.hotosm.org) and Humanitarian OpenStreetMap Team (HOT) (https://tasks.hotosm.org) task managers were used for the mapathons as the task managers allow us to divide up a mapping job into smaller tasks, and shows which areas need to be mapped and which areas have been mapped and need to be validated. At the end of each mapathon, the participants were invited to complete a questionnaire to determine their motivations and collect their personal opinions on the mapathon.

* Corresponding author 
Table 1. Overview of the four mapathons evaluated

\begin{tabular}{|c|c|c|c|c|c|}
\hline Mapathon & Date & Duration & Platform & Area mapped & Features mapped \\
\hline Mapathon1 & 2 March 2018 & $120 \mathrm{~min}$ & TeachOSM task & $\begin{array}{l}\text { Atteridgeville and Soshanguve, } \\
\text { City of Tshwane, South Africa }\end{array}$ & $\begin{array}{ll}- & \text { buildings } \\
- & \text { footpaths } \\
- & \text { roads }\end{array}$ \\
\hline Mapathon2 & $\begin{array}{l}\text { 23-24 March } \\
2018\end{array}$ & 7 hours & TeachOSM task & $\begin{array}{l}\text { L'Agulhas to Mossel Bay, } \\
\text { South Africa }\end{array}$ & $\begin{array}{ll}- & \text { boat launch site } \\
- & \text { cul-de-sac } \\
- & \text { car park } \\
- & \text { road } \\
- & \text { footpaths }\end{array}$ \\
\hline Mapathon3 & 18 May 2018 & $120 \mathrm{~min}$ & HOT task & $\begin{array}{l}\text { Kutupalong camp shelters, } \\
\text { Bangladesh }\end{array}$ & - $\quad$ buildings \\
\hline Mapathon4 & 21 May 2018 & $120 \mathrm{~min}$ & HOT task & $\begin{array}{l}\text { Kutupalong camp shelters, } \\
\text { Bangladesh }\end{array}$ & - $\quad$ buildings \\
\hline
\end{tabular}

\subsection{Participant motivation: Questionnaire}

To gather information about the participants' previous experience using OSM and their experience during the specific mapathon, we created a questionnaire that consisted of twelve questions using Qualtrics (https://www.qualtrics.com). The questions are listed in Table 2. The questionnaire covered various topics, such as describing a mapathon and the issues the participants' encountered. Some of the questions in the questionnaire were adapted from Ebrahim et al. (2016) that focused on mapping as a pedagogical tool for teaching 10-year-old children about geography. Even though these questions were designed for young children, we included them as the questions are suitable for understanding what the participants did not know before the mapathon (Table 2, question 7) and how they would describe a mapathon (Table 2, question 8).

The questionnaire was conducted at the end of all four mapathons (refer to Table 1) and all the responses were analysed. As the participants were recruited from the same pool, a large number of students participated in more than one mapathon. All responses for a participant were included and considered for the specific mapathon.

\subsection{Participant productivity: Data quality and quantity analysis}

The participants' productivity was measured in terms of the quantity of data contributed and the quality of the data. The analysis of the data was only done for Mapathon 1 and Mapathon 2, as these were TeachOSM tasks, specifically created for this project in areas that do not attract a lot of other mappers. The HOT tasks to which Mapathon 3 and Mapathon 4 contributed were linked to a recent disaster that attracted a large number of mappers. With tasks 3 and 4, the areas were mapped at a faster pace and other participants could have fixed errors our mapathon participants may have created. To assess the participants' productivity, the following two methods were used 1) the built-in validator in Java OpenStreetMap (JOSM) that uses the JTS Topology Suite; and 2) the raw OSM data including usernames, were downloaded and analysed in Desktop GIS software and PostgreSQL and PostGIS. The productivity of the participants was measured in terms of the number of edits per mapathon, and errors or warnings.

Table 2. Questionnaire on motivations and opinions.

\begin{tabular}{|c|c|c|}
\hline & $\begin{array}{c}\text { Question } \\
\end{array}$ & Options \\
\hline 1. & Are you familiar with the areas mapped during the mapathon? & Yes / No \\
\hline 2. & How many mapathons (including this one) have you attended? & \\
\hline 3. & Did you already have an OSM login before today? & Yes / No \\
\hline 4. & If yes, have you contributed data to OSM before today? & Yes / No \\
\hline 5 . & Do you think you have learned something by participating in mapathon? & Yes / No \\
\hline 6 . & If so, what is the most important thing you have learned? & Open-ended question \\
\hline 7. & \multicolumn{2}{|l|}{ What didn't you know before? } \\
\hline & $\circ \quad$ Humanitarian organisations need help & \multirow{5}{*}{$\begin{array}{l}\text { Multiple choice } \\
\text { question }\end{array}$} \\
\hline & $\circ \quad$ There are maps created by many people & \\
\hline & $\circ \quad$ Mapping is never finished work & \\
\hline & $\circ \quad$ Mapping the world is easy, fun and useful & \\
\hline & $\circ \quad$ Other, please specify & \\
\hline
\end{tabular}


Table 3. Questionnaire on motivations and opinions (continue)

\begin{tabular}{|c|c|c|}
\hline & Question & Options \\
\hline \multicolumn{3}{|c|}{ 8. How would you describe the mapathon? } \\
\hline & $\circ \quad$ Fun & \multirow{8}{*}{$\begin{array}{l}\text { Multiple answer } \\
\text { question, select all } \\
\text { that apply }\end{array}$} \\
\hline & Helpful/Useful & \\
\hline & Exciting & \\
\hline & Boring & \\
\hline & Interesting & \\
\hline & Useless & \\
\hline & Difficult & \\
\hline & $\circ \quad$ Other, please specify & \\
\hline 9. & How would you describe the mapathon to someone who does not know anything about it? & Open-ended question \\
\hline \multirow[t]{12}{*}{10.} & Rate your agreement with the following statements: & \multirow{12}{*}{$\begin{array}{l}\text { Strongly agree, } \\
\text { Agree, Neutral, } \\
\text { Disagree, Strongly } \\
\text { disagree }\end{array}$} \\
\hline & $\begin{array}{l}\text { a) I consider the data contributed during the mapathon to be of value for creating a base map to be } \\
\text { used by the target users }\end{array}$ & \\
\hline & I would participate in a mapathon in the future without any incentives offered & \\
\hline & $\begin{array}{l}\text { After completing this mapathon, I feel I am more 'in touch' with the community I mapped than } \\
\text { before }\end{array}$ & \\
\hline & $\begin{array}{l}\text { d) The social nature of the mapathon helped to create a more productive environment for capturing } \\
\text { data on OSM/HOT }\end{array}$ & \\
\hline & The social nature of the mapathon would persuade me to attend another mapathon & \\
\hline & I enjoyed using iD editor and capturing data on OSM/HOT & \\
\hline & I enjoyed the task we worked on today & \\
\hline & I would participate in a mapathon again only if incentives are offered & \\
\hline & Today's task was appropriate to teach me the basics of OSM and iD editor & \\
\hline & I found the iD editor intuitive and easy to use & \\
\hline & $\begin{array}{l}\text { After completing this mapathon, I will probably contribute data to OpenStreetMap (OSM) in my } \\
\text { own time by myself. }\end{array}$ & \\
\hline \multicolumn{2}{|r|}{ 11. What was the most difficult or challenging aspect of today's mapathon? } & \multirow{10}{*}{$\begin{array}{l}\text { Multiple answer } \\
\text { question, select all } \\
\text { that apply }\end{array}$} \\
\hline & $\circ \quad$ Bad aerial imagery or cloud cover & \\
\hline & It was difficult to learn to use iD editor at first & \\
\hline & It was difficult to identify roads and footpaths & \\
\hline & It was difficult to identify buildings & \\
\hline & It was difficult to trace the building footprint & \\
\hline & It was difficult to identify the type of road surface & \\
\hline & Slow internet connection & \\
\hline & Slow computers & \\
\hline & Lack of motivation due to Friday afternoon timeslot & \\
\hline 12. & Would you use OpenStreetMap (OSM) in the future? & Yes / No / Maybe \\
\hline
\end{tabular}

\section{RESULTS}

\subsection{Participants}

Students from the University of Pretoria, South Africa, enrolled for a final year geoinformatics module were invited to participate in the mapathon. Extra credit was offered to the students as an incentive. Table 3 provides an overview of the number of students and a breakdown of the gender distribution for the four mapathons.

Over the four mapathons, there were 60 students that participated in one or more mapathons. The majority of students $(60 \%)$ participated in at least two mapathons. Figure 1 provides an overview of the distribution. However, these students were invited to complete the questionnaire at each mapathon, and we received 115 responses from the 60 participants. The questionnaire was anonymous, therefore all 115 responses were evaluated for this paper, as the participants' thoughts on mapathons might change positively or negatively after completing more than one mapathon. The majority of the participants were in their third year of study in a degree related to geography (refer to Figure 2). This can be contributed to the limited pool from which the students were recruited.
Table 4. Participants of the four mapathons

\begin{tabular}{|c|c|c|c|c|}
\hline 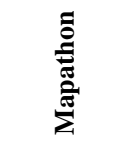 & 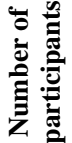 & $\sum^{\frac{0}{\pi}}$ & 节 & 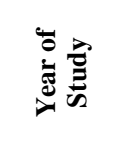 \\
\hline Mapathon1 & 36 & $\begin{array}{c}19 \\
(52.7 \%)\end{array}$ & $\begin{array}{c}17 \\
(47.3 \%)\end{array}$ & $\begin{array}{c}\text { 3rd years } \\
(100 \%)\end{array}$ \\
\hline Mapathon2 & 19 & $\begin{array}{c}8 \\
(42.1 \%)\end{array}$ & $\begin{array}{c}11 \\
(57.9 \%)\end{array}$ & $\begin{array}{c}\text { 3rd years } \\
(89.5 \%) \\
\text { 2nd years } \\
(10.5 \%)\end{array}$ \\
\hline Mapathon3 & 29 & $\begin{array}{c}17 \\
(58.6 \%)\end{array}$ & $\begin{array}{c}12 \\
(41.4 \%)\end{array}$ & $\begin{array}{c}\text { 3rd years } \\
(100 \%)\end{array}$ \\
\hline Mapathon4 & 31 & $\begin{array}{c}17 \\
(58.8 \%)\end{array}$ & $\begin{array}{c}14 \\
(45.2 \%)\end{array}$ & $\begin{array}{c}\text { 3rd years } \\
(100 \%)\end{array}$ \\
\hline
\end{tabular}




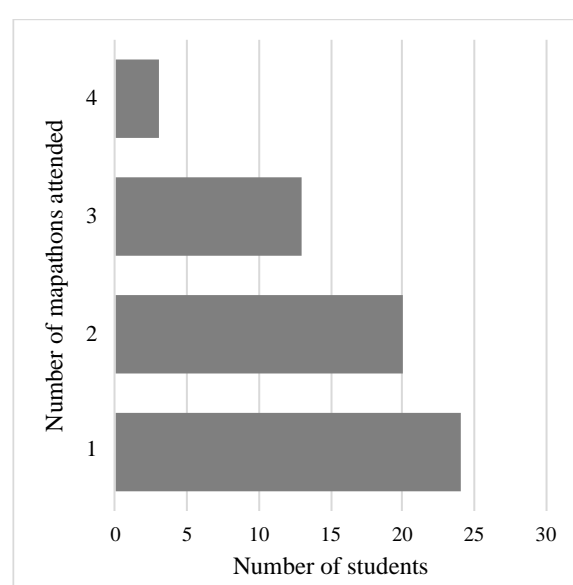

Figure 1. Number of mapathons students attended

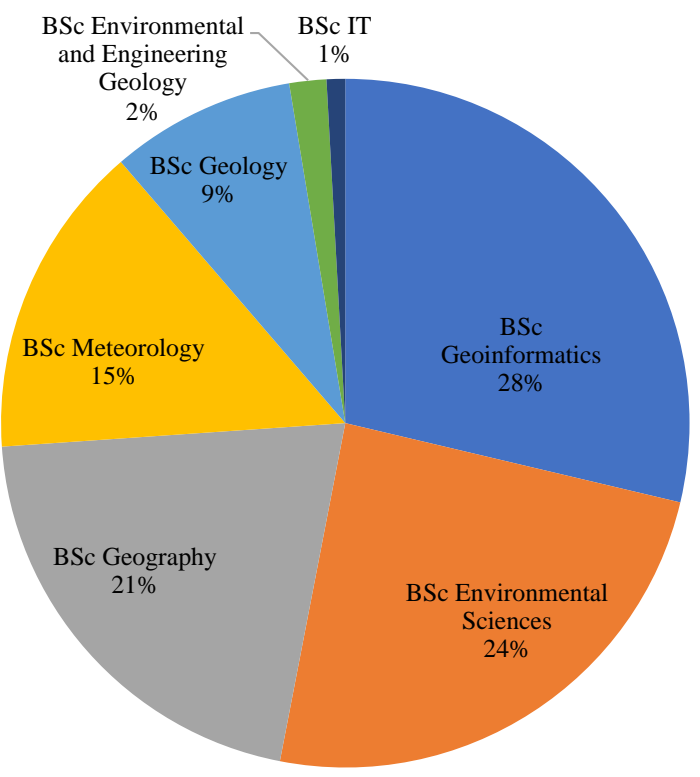

Figure 2. Degree programmes of participants

\subsection{Participant motivation: Questionnaire (Mapathon 1 - 4)}

\section{Participant retention}

The majority of the participants had not contributed to OSM before the first mapathon with only 2 participants indicating that they had an OSM account beforehand. This increased to $68 \%$ of participants at the second mapathon, indicating that the majority of the participants returned after the first mapathon. Referring also back to Figure 1, the majority $(60 \%)$ of the students participated in two or more mapathons. This is supported by the participants' average response to the statement in $10 . \mathrm{b}$ (refer to Table 2 and Figure 4) that they would participate in future mapathons without incentives, however, in statement 10.h (participate only when incentives are offered) the average response was neutral.
The participants were also asked if they would use OSM in future. $77 \%$ indicated that they would use OSM again and $21 \%$ indicated that they were not sure, with only $2 \%$ saying that they would not use OSM in the future.

\section{Describing a mapathon}

To better understand the participants' view of a mapathon, they were asked which words best describe a mapathon (refer to question 2 in Table 2 and Figure 3). 'Interesting' was the word most often selected, followed closely by 'helpful/useful' and 'fun'. This links closely to inherent characteristics of a mapathon being a teaching tool, altruistic and social. No participant selected useless, indicating that they understood the importance of the purpose of the mapathon and that the data they mapped will be used in real-world applications, for example, for mapathon 3 and 4 to deliver aid to refugees in Bangladesh.

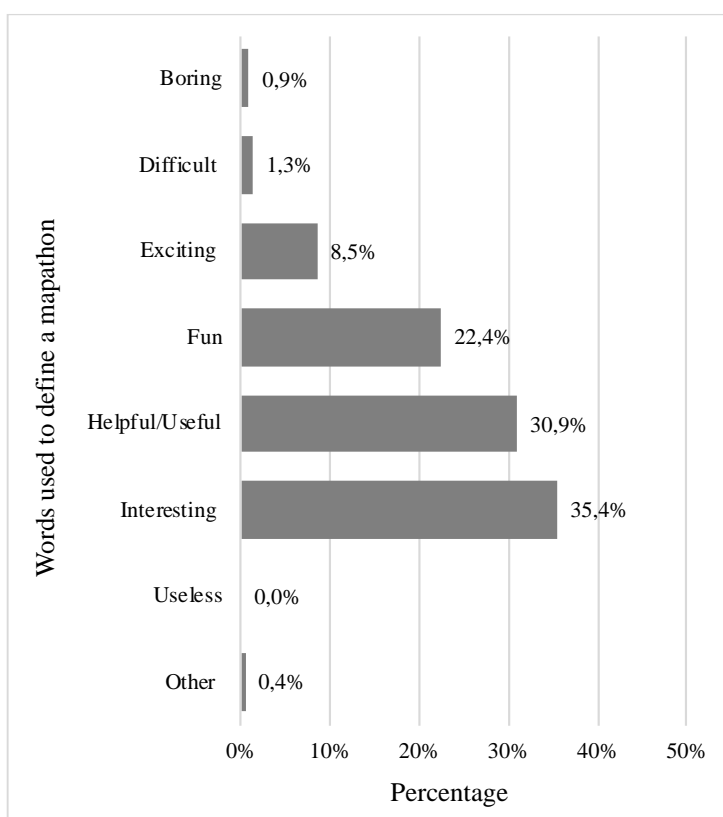

Figure 1. Words selected to describe a mapathon

Opinion on mapathons and OSM data editing

The participants were given a set of eleven statements and were asked to indicate their agreement with these using a five-point Likert scale (refer to question 10 in Table 2).

The participants' average agreement or disagreement with these statements over the four mapathons was calculated, see Figure 4. Generally, the participants agreed with all the statements, except for statement $\mathrm{c}, \mathrm{h}$ and $\mathrm{k}$. Interestingly, statement $h$ is the inverse of statement $b$ thus, even though the majority of the participants indicated that they would participate in mapathons again, it does seem like incentives are important. These incentives might not be extra credit but may be in the form of experience for their curriculum vitae. This is something that should be investigated further. Regarding statement $d$ and $\mathrm{k}$, the social aspect of mapathons seems to be the motivator for contributing to OSM. Lastly, from the responses, id Editor is clearly a good option for beginners and offers little to no barrier to entry. 


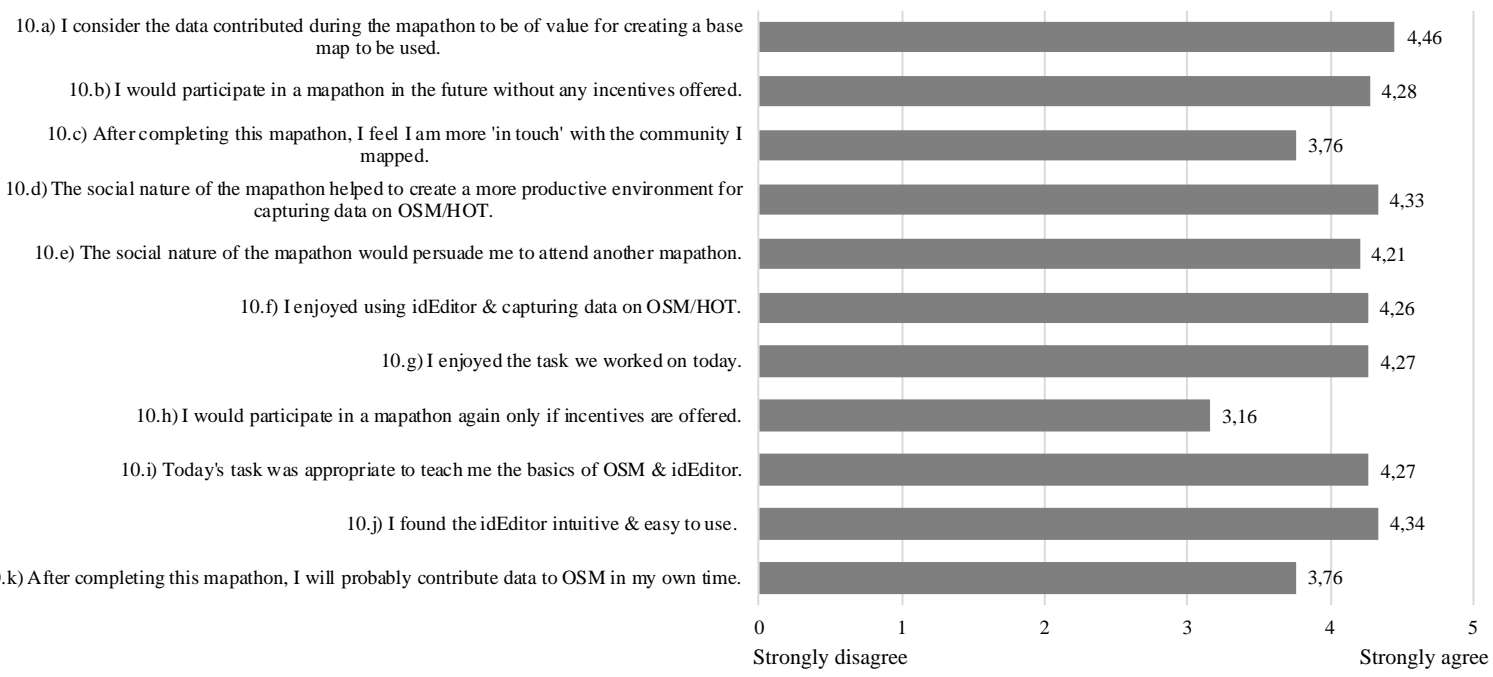

Figure 4. Participants' average agreement or disagreement with statement regarding their experience

Difficulties and challenges

The participants were asked to indicate if they encountered any challenges or issues during the mapathon. Refer to Figure 5. The biggest challenge encountered was bad aerial imagery or cloud cover (29\%), followed by identifying roads or footpaths $(20 \%)$ and identifying the road surface $(15.5 \%)$. These challenges were mainly linked to the second mapathon, as these were general comments and complaints during the mapathon.

The technology (i.e. internet speed, computers and id Editor) were not really identified as challenges. This can be attributed to the fact that the mapathon was in the computer lab of the university with good PCs and internet speed. Additionally, at each mapathon, there were at least two assistants in the venue to help the participants with any challenges.

What did the students learn?

The participants were asked if they learned something new during the mapathon. All participants indicated that they learned something new and were then asked to specify what they learned using free text. A word cloud was created with these responses using Wordle.net to highlight the words most often used. Refer to Figure 6. The topics the participants learned the most about were mapping and digitizing, and the importance of mapping for humanitarian purposes.

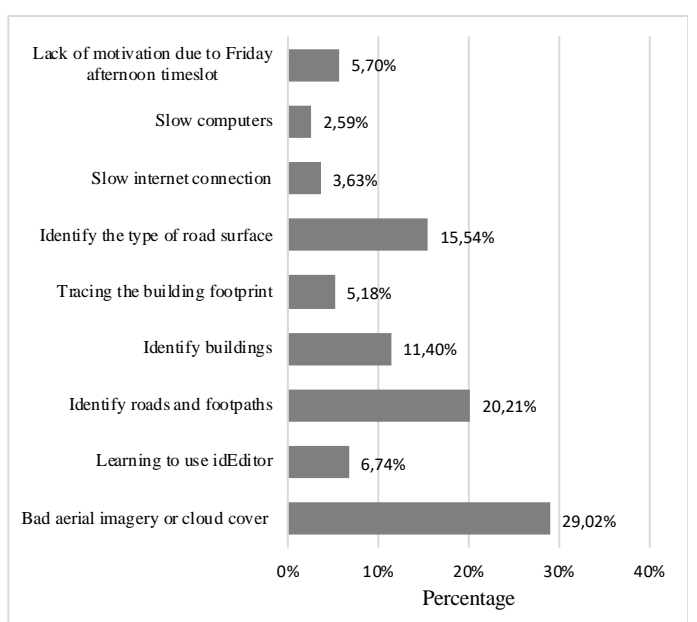

Figure 5. Difficulties and challenges encountered by participants

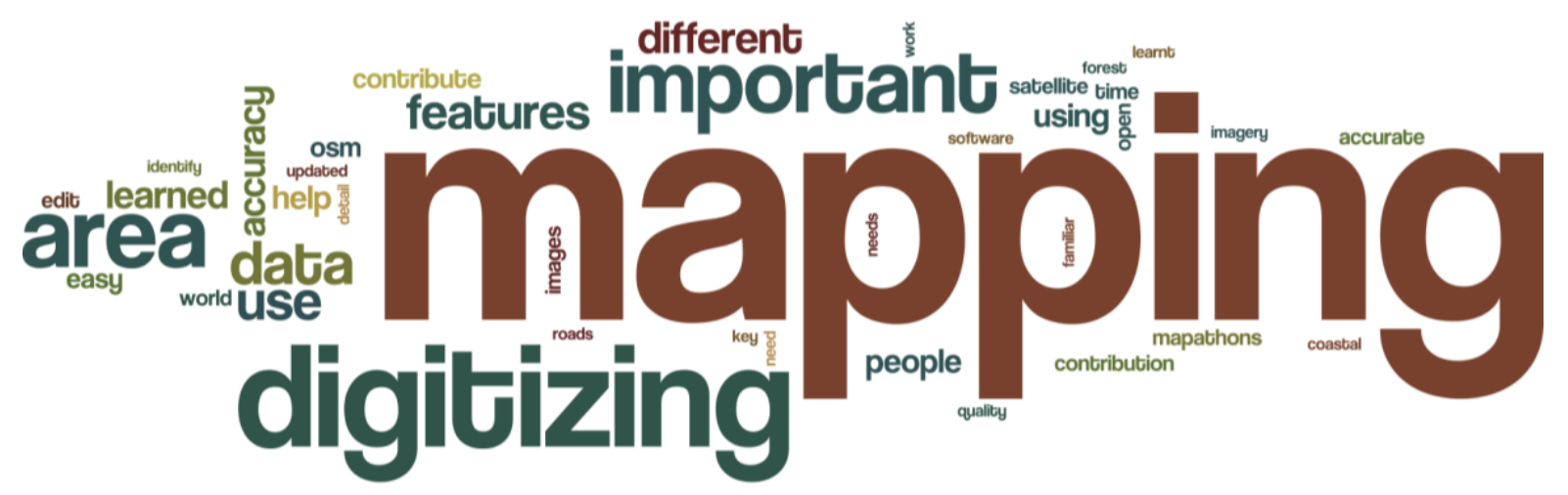

Figure 6. A word cloud on what participants indicated they learnt during the mapathon (created using http://www.wordle.net) 


\subsection{Participant productivity: Data quality and quantity analysis (Mapathon 1 and 2)}

The productivity was only calculated for mapathon 1 and mapathon 2, as these mapathons were focused on areas in South Africa and we could ensure that no external mappers corrected features mapped by our participants. Table 4 provides an overview of the areas mapped in these mapathons.

Table 4. Overview of mapathon productivity

\begin{tabular}{|c|c|c|}
\hline & Mapathon1 & Mapathon2 \\
\hline $\begin{array}{l}\text { Number of } \\
\text { participants }\end{array}$ & 36 participants & 19 participants \\
\hline Area mapped & $\begin{array}{l}\text { Atteridgeville and } \\
\text { Soshanguve, City } \\
\text { of Tshwane, South } \\
\text { Africa }\end{array}$ & $\begin{array}{l}\text { L'Agulhas to } \\
\text { Mossel Bay, } \\
\text { South Africa }\end{array}$ \\
\hline Size of area & $368.63 \mathrm{~km}^{2}$ & $6953.57 \mathrm{~km}^{2}$ \\
\hline Features mapped & $\begin{array}{l}\text { - buildings } \\
\text { - footpaths }\end{array}$ & $\begin{array}{l}\text { - roads } \\
\text { - boat launch site } \\
\text { - cul-de-sac } \\
\text { - car park } \\
\text { - road } \\
\text { - footpaths }\end{array}$ \\
\hline Duration & 90 minutes & $\begin{array}{l}420 \text { minutes ( } 7 \\
\text { hours) }\end{array}$ \\
\hline $\begin{array}{l}\text { Total number of } \\
\text { nodes mapped }\end{array}$ & 8242 nodes & 19953 nodes \\
\hline $\begin{array}{l}\text { Average nodes } \\
\text { mapped per } \\
\text { minute }\end{array}$ & 91.58 nodes & 47.51 nodes \\
\hline $\begin{array}{l}\text { Average nodes } \\
\text { per participant } \\
\text { Average }\end{array}$ & 305.26 nodes & 1050.16 nodes \\
\hline $\begin{array}{l}\text { Average } \\
\text { percentage of } \\
\text { nodes mapped } \\
\text { per participant }\end{array}$ & $3.7 \%$ & $5.26 \%$ \\
\hline
\end{tabular}

During mapathon 1, the area that was mapped was a dense urban area and the focus was on mapping the buildings and footpaths. Thus, it was very easy to find features to map, however, as it was the first mapathon the majority of the students completed the OSM editing walk though first before starting to work on the task. This might explain the low number of nodes mapped during this mapathon. However, for mapathon 2, due to the size of the area and the features mapped, the number of nodes mapped per minutes was lower than for the first mapathon. The participants needed to search through large natural areas to find these features and this took a lot of time.

The data mapped during these mapathons were validated using the JOSM validator. The validator checks for topological errors in the data, for example, is a road crossing a building or dangling node. The results from the JOSM validator found that there were more errors or warnings created when mapping the features for mapathon $1(4.32 \%)$ and only $1.22 \%$ for mapathon 2 . The majority of the errors or warnings were related to connecting footpaths to previously existing road segments. This can be tricky for beginners.

\section{DISCUSSION AND CONCLUSION}

In this paper, we presented the results from an evaluation of university students' motivation for participating in mapathons and their productivity. The results presented in Section 3 provided some unique insights into the participants' thoughts about and experiences during a mapathon, and OSM and related tools that can help organisers plan mapathons that would encourage mappers to produce quality data in OSM.

Although the participants indicated that they would participate in mapathons without incentive, volunteer retention in our study and related studies (Dittus 2016; Fritz et al. 2017) indicates that incentives are important. In this case, extra credit and in one case free a lunch was offered to the students, but there might be an alternative incentive that could be offered to participants. For example, HOT and YouthMappers offer students virtual internships that could be added to their curriculum vitae and serve as experience (Solis et al 2018; Fritz et al 2017). A virtual internship is a program where the participant gains work experience while working in a remote professional setting but is not physically present at the location.

The usefulness of OSM for potential future work, research projects or the general public seems to not be clear to all the participants as some participants indicated they would not use OSM in the future. The focus of the presentations before the mapathons has mainly been on the use of OSM for humanitarian purposes. Mapathon organisers should also arrange presentations that focus on a variety of uses of OSM. This will introduce the participants not only to the humanitarian uses of OSM, but also for other uses, such as for urban design which might also encourage one or two participants to use or contribute to OSM in the future. These presentations would also ensure that a mapathon has an interesting or helpful aspect to them, as this has been indicated as a highlight of mapathons.

The social and fun aspect of mapathons should continue to be encouraged (Coetzee et al. 2018; Fritz et al 2017; Liu et al 2017). For example, mapping competitions and music played during mapathons creates a fun environment that may encourage additional participants to attend. Mapathons can also be used to create an inclusive community within the student population and can help younger students when they start university to meet other students in their field.

The built-in editor in OSM, iD editor, was used for these mapathons. The majority of the participants indicated that $\mathrm{iD}$ editor is easy to use and intuitive. iD editor does not include advanced tools, such as validation, that might help the mapathon participants to produce higher quality data. iD editor is, however, a good tool for beginners and limits the barrier to entry, as it does not need to be installed (Vogler et al. 2017). After mappers have gained some experience, they could be introduced to advanced editors, such as JOSM. Editors, such as JOSM, would not only aid in limiting errors when mapping features but would also increase the mappers' productivity as it has the functionality to help with, for example adding and connecting road features.

The OSM wiki has useful information on adding tags to new features, but new mappers do not know about these resources. Additions to iD editor could potentially help new mappers select more appropriate tags (Ali et al. 2016; Vogler et al 2017). For example, providing a pop-up menu that would ask the mapper questions about what they see when they identify feature. These questions could then 
help the mapper to select the appropriate tags. This type of tool would need to be tested to confirm their usefulness for mapathons. Incorporating OSM into the curriculum of school and university has a pragmatic effect of introducing digitizing (i.e. a basic geospatial skill) while also contributing to society and improving their social responsibility (Coetzee et al. 2018; Solis et al. 2018; Hite et al. 2018; Mueller \& Solís, 2017).

The results from our surveys provide unique insight into the students' motivation for participating in mapathons and their productivity. These results and suggestions can be used by mapathon organisers to plan mapathons that would hopefully improve volunteer retention and encourage participants to produce high quality data.

\section{ACKNOWLEDGEMENTS}

The authors would like to thank the South African Geoinformatics Development Fund for the financial support which supported C. Green to attend the FOSS4G 2019 Conference in Bucharest.

\section{REFERENCES}

Ali, A., Sirilertworakul, N., Zipf, A., and Mobasheri, A., 2016. Guided Classification System for Conceptual Overlapping Classes in OpenStreetMap, ISPRS International Journal of Geo-Information, 5(6), pp. 87109.

Coetzee, S., Minghini, M., Solis, P., Rautenbach, V. and Green, C., 2018. Towards understanding the impact of Mapathons - Reflecting on YouthMappers experiences, The International Archives of the Photogrammetry, Remote Sensing and Spatial Information Sciences, Volume XLII-4/W8, 2018 FOSS4G 2018, 29-31 August 2018, Dar es Salaam, Tanzania.

Dittus, M., 2016. Large-scale Volunteer Engagement in Humanitarian Mapping. 19th ACM Conference on Computer Supported Cooperative Work and Social Computing Companion (CSCW '16), February 27-March 02, 2016, San Francisco, CA, USA. pp. 139-142.

Ebrahim, M., Minghini, M., Molinari, M.E., and Torrebruno, A., 2016. MiniMapathon - Mapping the world at 10 years old. Proceedings of the 8th annual International Conference on Education and New Learning Technologies (EDULEARN16), Barcelona (Spain), July 4-6, 2016, pp. 4200-4208.

Fritz, S., See, L., and Brovelli, M., 2017. Motivating and Sustaining Participation in VGI. In: Foody, G, See, L, Fritz, S, Mooney, P, Olteanu-Raimond, A-M, Fonte, C C and Antoniou, V. (eds.) Mapping and the Citizen Sensor. Pp. 93-117.

Hite, R., Solís, P., Wargo, L., and Larsen, T., 2018. Exploring Affective Dimensions of Authentic Geographic Education Using a Qualitative Document Analysis of Students' YouthMappers Blogs, Education Sciences, 8(4), p. 173-192.

Liu, H-Y., Grossberndt, S., and Kobernus, M., 2017. Citizen Science and Citizens' Observatories: Trends, Roles, Challenges and Development Needs for Science and Environmental Governance. In: Foody, G, See, L, Fritz, S, Mooney, P, Olteanu- Raimond, A-M, Fonte, C C and Antoniou, V. (eds.) Mapping and the Citizen Sensor. Pp. 351-376.

Mueller, T., and Solís, P., 2017. Humanitarian Mapping Education: A View on Curriculum. Humanitarian Open Street Map Team Summit. Ottawa, Canada, September 1415 September.

Solís, P. McCusker, B., Menkiti, N., Cowan, N., Blevins, C. 2018. Engaging global youth in participatory spatial data creation for the UN sustainable development goals: The case of open mapping for malaria prevention, Applied Geography, 98, pp. 143-155.

Vogler, R., Hennig, S. and Ferber, N., 2017. Redressing the Exclusiveness. Challenges which Prevent New Users from Contributing to OSM., GI Forum (2), pp. 113-125. 